\title{
Multiplicity results for systems of first order dynamic inclusions on time scales
}

\author{
Marlène Frigon ${ }^{1 *}$ (i) and Maryam Lotfipour²
}

\section{"Correspondence:}

frigon@dms.umontreal.ca

'Département de Mathématiques

et de Statistique, Université de

Montréal, Montréal, H3C 3J7,

Canada

Full list of author information is

available at the end of the article

\begin{abstract}
Multiplicity results are obtained for systems of first order dynamic inclusions on time scales with the periodic boundary condition. To this aim, we introduce the notion of a strict solution-tube. We consider the case where the nonlinearity satisfies an upper semi-continuity condition. Our results are new even in the particular cases where the nonlinearity is single-valued or it has real values.
\end{abstract}

MSC: Primary 34N05; secondary 34A60; 39A23

Keywords: system of dynamic inclusions; time scale; first order problem; multiple solutions; solution-tube; strict solution-tube

\section{Introduction}

The concept of dynamic equations on time scales was initiated by Hilger [1] in 1990. By introducing the notion of delta-derivative $x^{\Delta}(t)$, which coincides with $x^{\prime}(t)$ (resp. $\left.\Delta x(t)\right)$ in the case where the time scale $\mathbb{T}$ is an interval (resp. a discrete set $\{0,1, \ldots, n\}$ ), a unified approach to discrete and continuous calculus is provided. In this way, one can easily carry over many results concerning differential equations to corresponding results for difference equations. This can avoid proving results separately.

In this paper, we establish multiplicity results for the following system of first order dynamic inclusions:

$$
\begin{aligned}
& x^{\Delta}(t) \in F(t, x(\sigma(t))), \quad \Delta \text {-a.e. } t \in \mathbb{T}_{0}, \\
& x(a)=x(b) .
\end{aligned}
$$

Here $\mathbb{T}$ is an arbitrary compact time scale such that $a=\min \mathbb{T}$ and $b=\max \mathbb{T}, \mathbb{T}_{0}=\mathbb{T} \backslash\{b\}$, and $F: \mathbb{T}_{0} \times \mathbb{R}^{n} \rightarrow \mathbb{R}^{n}$ is a set-valued mapping with compact, convex values which satisfies an upper semi-continuity condition.

In the particular case where $n=1$, first existence results for this type of problems on an arbitrary compact time scale were obtained by Atici and Biles [2]. Their results were established with the method of lower and upper solutions.

Existence results were obtained in [3-6] for systems of dynamic equations on time scales (that is, for $n>1$ and in the particular case where $F$ is single-valued). In particular, a kind of coordinate-wise lower and upper solutions condition was imposed in [4], while Gilbert [5]

(c) 2015 Frigon and Lotfipour. This article is distributed under the terms of the Creative Commons Attribution 4.0 International License (http://creativecommons.org/licenses/by/4.0/), which permits unrestricted use, distribution, and reproduction in any medium, provided you give appropriate credit to the original author(s) and the source, provide a link to the Creative Commons license, and indicate if changes were made. 
introduced the notion of solution-tube to systems of first order dynamic equations which generalizes the notions of lower and upper solutions.

Existence results for systems of first order dynamic inclusions were obtained in $[7,8]$ for the initial value problem (that is, problem (1.1) with $n>1, F$ multi-valued, and the periodic boundary condition replaced by $x(a)=x_{0}$ ). Frigon and Gilbert [9] were the first to give conditions insuring the existence of a solution to the system (1.1) with the periodic boundary condition. To this aim, they extended the notion of solution-tube to systems of first order dynamic inclusions on time scales. It is worth to mention that the concept of solution-tube generalizes the notion of lower and upper solutions given in [2].

In the literature, there are very few results establishing the existence of more than one solution to first order problems. Sun and Li [10] established the existence of at least three solutions of (1.1) in the particular case where $n=1$ and $F$ is single-valued. In the particular case where $n>1$ and $\mathbb{T}=[a, b]$, problem (1.1) is in fact a system of first order differential inclusions. Multiplicity results were obtained by the authors in [11] for such systems of differential inclusions.

To our knowledge, in this paper, we establish the first multiplicity result for the system (1.1). In order to obtain the existence of at least three solutions of (1.1), we introduce the notion of a strict solution-tube of (1.1). It is inspired by a notion introduced in [11] for systems of first order differential inclusions. It is worth to mention that our result are new even in the particular cases where the nonlinearity is single-valued or it has real values.

\section{Preliminaries}

In this section, we recall some notions and results which we will use in this article.

\subsection{Multi-valued maps and topological degree}

For the sake of completeness, we recall some definitions in the theory of multi-valued mappings. Let $X$ and $Y$ be topological spaces, and $Z$ a measurable space. We say that a set-valued mapping $F: X \rightarrow Y$ is compact if $F(X)=\bigcup_{x \in X} F(x)$ is relatively compact, and $F$ is upper semi-continuous (u.s.c.) if $\{x \in X: F(x) \cap C \neq \emptyset\}$ is closed for every closed set $C \subset Y$. We say that a set-valued mapping $F: Z \rightarrow Y$ is measurable if $\{z \in Z: F(z) \cap C \neq \emptyset\}$ is measurable for each closed set $C \subset Y$. The reader is referred to [12-14] for more details on set-valued mappings.

Our results will rely on the topological degree theory for multi-valued maps. We recall some properties. The interested reader can consult [15]. Let $E$ be a real Banach space. We denote by $\mathcal{A}$ the set of pairs $(\Omega$,id $-F)$ such that $\Omega \subset E$ is a nonempty, open, bounded set, and $F: \bar{\Omega} \rightarrow E$ is a compact, u.s.c. multi-valued map with nonempty, compact, convex values and such that $x \notin F(x)$ for every $x \in \partial \Omega$. The topological degree on $E$ for multi-valued maps associates to every $(\Omega, \mathrm{id}-F) \in \mathcal{A}$ an integer $\operatorname{deg}(\mathrm{id}-F, \Omega)$ satisfying the following properties:

(i) (Normalization) If $0 \in \Omega, \operatorname{deg}(\mathrm{id}, \Omega)=1$, for $y \in \Omega$.

(ii) (Additivity) If $\Omega_{1}, \Omega_{2}$ are disjoint, open subsets of $\Omega$ such that $0 \notin(\mathrm{id}-F)\left(\bar{\Omega} \backslash \Omega_{1} \cup \Omega_{2}\right)$, then

$$
\operatorname{deg}(\mathrm{id}-F, \Omega)=\operatorname{deg}\left(\mathrm{id}-F, \Omega_{1}\right)+\operatorname{deg}\left(\mathrm{id}-F, \Omega_{2}\right) .
$$

(iii) (Homotopy) If $H:[0,1] \times \bar{\Omega} \rightarrow E$ is a compact, u.s.c. multi-valued map with nonempty, compact, convex values such that $0 \notin(\mathrm{id}-H(t, \cdot))(\partial \Omega)$ for every 
$t \in[0,1]$, then

$$
\operatorname{deg}(\mathrm{id}-H(t, \cdot), \Omega)=\operatorname{deg}(\mathrm{id}-H(0, \cdot), \Omega) \quad \forall t \in[0,1] .
$$

(iv) (Existence) If $\operatorname{deg}(\mathrm{id}-F, \Omega) \neq 0$, then there exists $x \in \Omega$ such that $x \in F(x)$.

(v) (Excision) If $\Omega_{1} \subset \Omega$ is open and $0 \notin(\mathrm{id}-F)\left(\bar{\Omega} \backslash \Omega_{1}\right)$, then

$$
\operatorname{deg}(\mathrm{id}-F, \Omega)=\operatorname{deg}\left(\mathrm{id}-F, \Omega_{1}\right) .
$$

\subsection{Time scale and $\Delta$-measure}

Let $\mathbb{T}$ be a compact time scale with

$$
a=\min \mathbb{T}<b=\max \mathbb{T} .
$$

The forward jump operator $\sigma: \mathbb{T} \rightarrow \mathbb{T}$ (resp. the backward jump operator $\rho: \mathbb{T} \rightarrow \mathbb{T}$ ) is defined by

$$
\begin{gathered}
\sigma(t)= \begin{cases}\inf \{s \in \mathbb{T}: s>t\} & \text { if } t<b, \\
b & \text { if } t=b\end{cases} \\
\left(\text { resp. } \rho(t)=\left\{\begin{array}{ll}
\sup \{s \in \mathbb{T}: s<t\} & \text { if } t>a, \\
a & \text { if } t=a
\end{array}\right) .\right.
\end{gathered}
$$

We say that $t<b$ is right-scattered (resp. $t>a$ is left-scattered) if $\sigma(t)>t$ (resp. $\rho(t)<t)$. The set of right-scattered points of $\mathbb{T}$ is at most countable; see [16]. We denote it by

$$
R_{\mathbb{T}}=\{t \in \mathbb{T}: t<\sigma(t)\}=\left\{t_{i}: i \in I\right\}
$$

for some $I \subset \mathbb{N}$. The graininess function $\mu: \mathbb{T} \rightarrow[0, \infty)$ is defined by $\mu(t)=\sigma(t)-t$. We denote

$$
\mathbb{T}_{0}=\mathbb{T} \backslash\{b\}
$$

Definition 2.1 A map $f: \mathbb{T} \rightarrow \mathbb{R}^{n}$ is $\Delta$-differentiable at $t \in \mathbb{T} \backslash(\rho(b), b]$ if there exists $f^{\Delta}(t) \in \mathbb{R}^{n}$ (called the $\Delta$-derivative of $f$ at $t$ ) such that for all $\varepsilon>0$, there exists a neighborhood $U$ of $t$ such that

$$
\left\|\left(f(\sigma(t))-f(s)-f^{\Delta}(t)(\sigma(t)-s)\right)\right\| \leq \varepsilon|\sigma(t)-s| \quad \forall s \in U .
$$

We say that $f$ is $\Delta$-differentiable if $f^{\Delta}(t)$ exists for every $t \in \mathbb{T} \backslash(\rho(b), b]$.

Definition 2.2 A function $f: \mathbb{T} \rightarrow \mathbb{R}^{n}$ is called $r d$-continuous provided it is continuous at right-dense points of $\mathbb{T}$ and its left-sided limits exist at left-dense points of $\mathbb{T}$. The set of rd-continuous functions $f: \mathbb{T} \rightarrow \mathbb{R}^{n}$ is denoted by $C_{r d}\left(\mathbb{T}, \mathbb{R}^{n}\right)$. The set of functions $f:$ $\mathbb{T} \rightarrow \mathbb{R}^{n}$ which are $\Delta$-differentiable and whose $\Delta$ - derivative is rd-continuous is denoted by $C_{r d}^{1}\left(\mathbb{T}, \mathbb{R}^{n}\right)$ and, moreover,

$$
C_{0, r d}^{1}(\mathbb{T})=\left\{\phi: \mathbb{T} \rightarrow \mathbb{R}: \phi \in C_{r d}^{1}(\mathbb{T}, \mathbb{R}) \text { and } \phi(a)=0=\phi(b)\right\} .
$$


We recall some notions and results related to the theory of a $\Delta$-measure.

Definition 2.3 ([17]) A set $A \subset \mathbb{T}$ is said to be $\Delta$-measurable if, for every set $E \subset \mathbb{T}$,

$$
m_{1}^{*}(E)=m_{1}^{*}(E \cap A)+m_{1}^{*}(E \cap(\mathbb{T} \backslash A))
$$

where

$$
m_{1}^{*}(E)= \begin{cases}\inf \left\{\sum_{k=1}^{m}\left(d_{k}-c_{k}\right): E \subset \bigcup_{k=1}^{m}\left[c_{k}, d_{k}\right) \text { with } c_{k}, d_{k} \in \mathbb{T}\right\} & \text { if } b \notin E, \\ \infty & \text { if } b \in E .\end{cases}
$$

The $\Delta$-measure on $\mathcal{M}\left(m_{1}^{*}\right)=\{A \subset \mathbb{T}: A$ is $\Delta$-measurable $\}$, denoted by $\mu_{\Delta}$, is the restriction of $m_{1}^{*}$ to $\mathcal{M}\left(m_{1}^{*}\right)$. So, $\left(\mathbb{T}, \mathcal{M}\left(m_{1}^{*}\right), \mu_{\Delta}\right)$ is a complete measurable space.

Proposition 2.4 ([16]) Let $A \subset \mathbb{T}$. Then $A$ is $\Delta$-measurable if and only if $A$ is Lebesgue measurable.

Inspired by Proposition 4.1 in [16], we prove the following proposition.

Proposition 2.5 Let $F: \mathbb{T}_{0} \rightarrow \mathbb{R}^{n}$ be a set-valued mapping and $\widetilde{F}:[a, b) \rightarrow \mathbb{R}^{n}$ defined by

$$
\widetilde{F}(t)= \begin{cases}F(t) & \text { if } t \in \mathbb{T}_{0}, \\ F\left(t_{i}\right) & \text { if } t \in\left(t_{i}, \sigma\left(t_{i}\right)\right), \text { for some } i \in I\end{cases}
$$

Then $F$ is $\Delta$-measurable if and only if $\widetilde{F}$ is Lebesgue measurable.

Proof Let $C \subset \mathbb{R}$ be a closed set. One has

$$
\begin{aligned}
\{t & \in[a, b): \widetilde{F}(t) \cap C \neq \emptyset\} \\
& =\left\{t \in \mathbb{T}_{0}: \widetilde{F}(t) \cap C \neq \emptyset\right\} \cup\left\{t \in \bigcup_{i \in I}\left(t_{i}, \sigma\left(t_{i}\right)\right): \widetilde{F}(t) \cap C \neq \emptyset\right\} \\
& =\left\{t \in \mathbb{T}_{0}: F(t) \cap C \neq \emptyset\right\} \cup\left(\bigcup_{i \in K}\left(t_{i}, \sigma\left(t_{i}\right)\right)\right),
\end{aligned}
$$

where $K=\left\{i \in I: F\left(t_{i}\right) \cap C \neq \emptyset\right\}$.

Since $F$ is $\Delta$-measurable, $I$ is at most countable and using Proposition 2.4, we deduce the conclusion.

Let $E \subset \mathbb{T}$ be a $\Delta$-measurable set and $f: \mathbb{T} \rightarrow \mathbb{R}^{n}$ be a $\Delta$-measurable function. We say that $f \in L_{\Delta}^{1}\left(E, \mathbb{R}^{n}\right)$ provided

$$
\int_{E}\|f(s)\| \Delta s<\infty
$$

The set $L_{\Delta}^{1}\left(\mathbb{T}_{0}, \mathbb{R}^{n}\right)$ is a Banach space endowed with the norm

$$
\|f\|_{L_{\Delta}^{1}}=\int_{\mathbb{T}_{0}}\|f(s)\| \Delta s
$$


We also recall the notion of a Sobolev space, see [18]:

$$
\begin{aligned}
W_{\Delta}^{1,1}\left(\mathbb{T}, \mathbb{R}^{n}\right)= & \left\{x \in L_{\Delta}^{1}\left(\mathbb{T}_{0}, \mathbb{R}^{n}\right): \exists g \in L_{\Delta}^{1}\left(\mathbb{T}_{0}, \mathbb{R}^{n}\right)\right. \text { such that } \\
& \left.\int_{\mathbb{T}_{0}} x(s) \phi^{\Delta}(s) \Delta s=-\int_{\mathbb{T}_{0}} g(s) \phi(\sigma(s)) \Delta s, \forall \phi \in C_{0, r d}^{1}(\mathbb{T})\right\} .
\end{aligned}
$$

If $x \in W_{\Delta}^{1,1}\left(\mathbb{T}, \mathbb{R}^{n}\right)$, then $x$ is $\Delta$-differentiable $\Delta$-a.e. on $\mathbb{T}_{0}$ and $x^{\Delta}$ is the function $g$ given in the definition of $W_{\Delta}^{1,1}\left(\mathbb{T}, \mathbb{R}^{n}\right)$.

In order to obtain a priori bounds for solutions to suitable family of problems, we will use the following result due to Gilbert [5].

Lemma 2.6 Let $s \in W_{\Delta}^{1,1}(\mathbb{T}, \mathbb{R})$ be such that

$$
s^{\Delta}(t)<0, \quad \Delta \text {-a.e. } t \in\left\{t \in \mathbb{T}_{0}: s(\sigma(t))>0\right\} .
$$

If one of the following conditions holds:

(i) $s(a) \leq 0$;

(ii) $s(a) \leq s(b)$;

then $s(t) \leq 0$ for every $t \in \mathbb{T}$.

We recall the following existence result for dynamical systems of linear equations on time scales, which is based on the exponential function $e_{1}(\cdot, a)$ defined by

$$
e_{1}(t, a)=\exp \left(\int_{a}^{t} \xi_{1}(\mu(s)) \Delta s\right)
$$

where

$$
\xi_{1}(h)= \begin{cases}1 & \text { if } h=0, \\ \frac{\log (1+h)}{h} & \text { if } h>0 .\end{cases}
$$

The interested reader can find the proof of the following proposition in [5] (see also [3, 6] for the continuous case).

Proposition 2.7 For every $g \in L_{\Delta}^{1}\left(\mathbb{T}_{0}, \mathbb{R}^{n}\right)$, the problem

$$
\begin{aligned}
& x^{\Delta}(t)+x(\sigma(t))=g(t), \quad \Delta \text {-a.e. } t \in \mathbb{T}_{0}, \\
& x(a)=x(b)
\end{aligned}
$$

has a unique solution $x \in W_{\Delta}^{1,1}\left(\mathbb{T}, \mathbb{R}^{n}\right)$ given by

$$
x(t)=\frac{1}{e_{1}(t, a)}\left(\frac{1}{e_{1}(b, a)-1} \int_{[a, b) \cap \mathbb{T}} g(s) e_{1}(s, a) \Delta s+\int_{[a, t) \cap \mathbb{T}} g(s) e_{1}(s, a) \Delta s\right) .
$$

Moreover, this defines a continuous linear operator $L: L_{\Delta}^{1}\left(\mathbb{T}_{0}, \mathbb{R}^{n}\right) \rightarrow C\left(\mathbb{T}, \mathbb{R}^{n}\right)$ by $L(g)=x$ given in (2.3). 


\section{3 $\Delta$-Carathéodory multi-valued maps}

Definition 2.8 A set-valued mapping $F: \mathbb{T}_{0} \times \mathbb{R}^{n} \rightarrow \mathbb{R}^{n}$ with nonempty closed, convex values is said to be $\Delta$-Carathéodory if the following conditions hold:

(i) $t \mapsto F(t, x)$ is $\Delta$-measurable for every $x \in \mathbb{R}^{n}$;

(ii) $x \mapsto F(t, x)$ is u.s.c. for $\Delta$-a.e. $t \in \mathbb{T}_{0}$;

(iii) for every $r>0$, there exists $h_{r} \in L_{\Delta}^{1}\left(\mathbb{T}_{0}, \mathbb{R}\right)$ such that

$$
\sup \{\|y\|: y \in F(t, x),\|x\| \leq r\} \leq h_{r}(t), \quad \Delta \text {-a.e. } t \in \mathbb{T}_{0} .
$$

Remark 2.9 A single-valued mapping $f: \mathbb{T}_{0} \times \mathbb{R}^{n} \rightarrow \mathbb{R}^{n}$ is $\Delta$-Carathéodory if and only if $F=\{f\}$ is $\Delta$-Carathéodory in the sense of Definition 2.8.

The mappings in the next two examples will be useful later.

Example 2.10 Let $(\nu, r) \in W_{\Delta}^{1,1}\left(\mathbb{T}, \mathbb{R}^{n}\right) \times W_{\Delta}^{1,1}(\mathbb{T},(0, \infty))$, and let the multi-valued map $G_{(v, r)}: \mathbb{T}_{0} \times \mathbb{R}^{n} \rightarrow \mathbb{R}^{n}$ be defined by

$$
G_{(v, r)}(t, x)= \begin{cases}\left\{z \in \mathbb{R}^{n}:\left\langle x-v(\sigma(t)), z-v^{\Delta}(t)\right\rangle\right. & \\ \left.\leq r^{\Delta}(t)\|x-v(\sigma(t))\|\right\} & \text { if } r(\sigma(t))<\|x-v(\sigma(t))\|, \\ \mathbb{R}^{n} & \text { otherwise. }\end{cases}
$$

Then, arguing as in [9], one can show that $G_{(v, r)}$ has nonempty, closed, convex values and it satisfies the following properties:

(i) $t \mapsto G_{(v, r)}(t, x)$ is $\Delta$-measurable for all $x \in \mathbb{R}^{n}$;

(ii) $x \mapsto G_{(v, r)}(t, x)$ is u.s.c. for $\Delta$-a.e. $t \in \mathbb{T}_{0}$.

Example 2.11 Let $(v, r) \in W_{\Delta}^{1,1}\left(\mathbb{T}, \mathbb{R}^{n}\right) \times W_{\Delta}^{1,1}(\mathbb{T},(0, \infty)), \varepsilon: \mathbb{T} \rightarrow(0, \infty)$ a lower semicontinuous (l.s.c.) single-valued mapping, and let $K_{(v, r, \varepsilon)}: \mathbb{T}_{0} \times \mathbb{R}^{n} \rightarrow \mathbb{R}^{n}$ be defined by

$$
K_{(v, r, \varepsilon)}(t, x)=\left\{\begin{array}{cc}
\left\{z \in \mathbb{R}^{n}:\left\langle x-v(\sigma(t)), z-v^{\Delta}(t)\right\rangle\right. & \\
\left.\leq r^{\Delta}(t)\|x-v(\sigma(t))\|\right\} & \text { if } r(\sigma(t))-\varepsilon(\sigma(t)) \\
& <\|x-v(\sigma(t))\|<r(\sigma(t)), \\
\mathbb{R}^{n} & \text { otherwise. }
\end{array}\right.
$$

Then, arguing as in [11], it can be shown that $K_{(v, r, \varepsilon)}$ has nonempty, closed, convex values and it has the following properties:

(i) $t \mapsto K_{(v, r, \varepsilon)}(t, x)$ is $\Delta$-measurable for all $x \in \mathbb{R}^{n}$;

(ii) $x \mapsto K_{(v, r, \varepsilon)}(t, x)$ is u.s.c. for $\Delta$-a.e. $t \in \mathbb{T}_{0}$.

The following proposition is an easy consequence of well-known properties of setvalued mappings.

Proposition 2.12 Let $M_{0}, M_{1}: \mathbb{T}_{0} \times \mathbb{R}^{n} \rightarrow \mathbb{R}^{n}$ be multi-valued maps with nonempty, closed, convex values such that

(i) $M_{0}$ is $\Delta$-Carathéodory; 
(ii) $t \mapsto M_{1}(t, x)$ is $\Delta$-measurable for all $x \in \mathbb{R}^{n}$, and $x \mapsto M_{1}(t, x)$ is u.s.c.for $\Delta$-a.e.

$$
t \in \mathbb{T}_{0}
$$

(iii) $M_{0}(t, x) \cap M_{1}(t, x) \neq \emptyset$ for all $x \in \mathbb{R}^{n}$ and $\Delta$-a.e. $t \in \mathbb{T}_{0}$.

Then $M_{0} \cap M_{1}$ is $\Delta$-Carathéodory.

For $(v, r) \in W_{\Delta}^{1,1}\left(\mathbb{T}, \mathbb{R}^{n}\right) \times W_{\Delta}^{1,1}(\mathbb{T},(0, \infty))$, we denote the projection of $x$ on the closed ball centered in $v(t)$ of radius $r(t)$ by

$$
\bar{x}_{(v, r)}(t, x)= \begin{cases}\frac{r(t)}{\|x-v(t)\|}(x-v(t))+v(t) & \text { if }\|x-v(t)\|>r(t) \\ x & \text { otherwise. }\end{cases}
$$

For a set-valued mapping $F: \mathbb{T}_{0} \times \mathbb{R}^{n} \rightarrow \mathbb{R}^{n}$, we define $\mathcal{F}: C\left(\mathbb{T}, \mathbb{R}^{n}\right) \rightarrow C\left(\mathbb{T}, \mathbb{R}^{n}\right)$ by

$$
\mathcal{F}(x)=L\left(\left\{y \in L_{\Delta}^{1}\left(\mathbb{T}_{0}, \mathbb{R}^{n}\right): y(t) \in F(t, x(\sigma(t))), \Delta \text {-a.e. } t \in \mathbb{T}_{0}\right\}\right)
$$

where $L$ is the function defined in Proposition 2.7.

Fixed points of such a map will permit us to obtain solutions to our system of first order dynamic inclusions. In the following result, which will be used later, a particular map $F$ is considered and conditions are given ensuring that the associated map $\mathcal{F}$ is a compact, u.s.c. multi-valued map with nonempty, convex, compact values.

Proposition 2.13 Let $M_{0}, M_{1}: \mathbb{T}_{0} \times \mathbb{R}^{n} \rightarrow \mathbb{R}^{n}$ be multi-valued maps with nonempty, closed, convex values, and let $(\nu, r) \in W_{\Delta}^{1,1}\left(\mathbb{T}, \mathbb{R}^{n}\right) \times W_{\Delta}^{1,1}(\mathbb{T},(0, \infty))$. We assume that

(i) $M_{0}$ is $\Delta$-Carathéodory;

(ii) $t \mapsto M_{1}(t, x)$ is $\Delta$-measurable for all $x \in \mathbb{R}^{n}$, and $x \mapsto M_{1}(t, x)$ is u.s.c.for $\Delta$-a.e. $t \in \mathbb{T}_{0}$

(iii) $\Delta$-a.e. $t \in \mathbb{T}_{0}$ and for every $x \in C\left(\mathbb{T}, \mathbb{R}^{n}\right)$,

$$
M_{0}\left(t, \bar{x}_{(v, r)}(\sigma(t), x(\sigma(t)))\right) \cap M_{1}(t, x(\sigma(t))) \neq \emptyset
$$

Let $F: \mathbb{T}_{0} \times \mathbb{R}^{n} \rightarrow \mathbb{R}^{n}$ be defined by

$$
F(t, x)=\bar{x}_{(v, r)}(\sigma(t), x)+M_{0}\left(t, \bar{x}_{(v, r)}(\sigma(t), x)\right) \cap M_{1}(t, x)
$$

and let $\mathcal{F}: C\left(\mathbb{T}, \mathbb{R}^{n}\right) \rightarrow C\left(\mathbb{T}, \mathbb{R}^{n}\right)$ be the multi-valued map associated to $F$ given in (2.7). Then $\mathcal{F}$ is u.s.c., compact with nonempty, convex, compact values.

The proof of this result follows from arguments analogous to those used in Proposition 3.5 in [9], or using Proposition 2.5 and arguing as in Proposition 3.4 in [19] or Proposition 3.5 in [11].

\section{Multiplicity results}

In this section, we establish a multiplicity result for the system of first order dynamic inclusions (1.1). To this aim, we use the following slight modification of the notion of solutiontube introduced in [9]. 
Definition 3.1 Let $(v, r) \in W_{\Delta}^{1,1}\left(\mathbb{T}, \mathbb{R}^{n}\right) \times W_{\Delta}^{1,1}(\mathbb{T},(0, \infty))$. We say that $(v, r)$ is a solutiontube of (1.1) if

(i) $\Delta$-a.e. $t \in \mathbb{T}_{0}$ and for every $x \in \mathbb{R}^{n}$ such that $\|x-v(\sigma(t))\|=r(\sigma(t))$, there exists $y \in F(t, x)$ such that

$$
\left\langle x-v(\sigma(t)), y-v^{\Delta}(t)\right\rangle \leq r(\sigma(t)) r^{\Delta}(t)
$$

(ii) $\|v(b)-v(a)\| \leq r(a)-r(b)$.

We denote

$$
T(v, r)=\left\{x \in C\left(\mathbb{T}, \mathbb{R}^{n}\right):\|x(t)-v(t)\| \leq r(t) \text { for every } t \in \mathbb{T}\right\}
$$

It was shown in [9] that the existence of a solution-tube ensures the existence of a solution of (1.1).

Theorem 3.2 ([9]) Let $F: \mathbb{T}_{0} \times \mathbb{R}^{n} \rightarrow \mathbb{R}^{n}$ be a Carathéodory set-valued mapping with nonempty, closed, convex values. Assume there exists $(v, r) \in W_{\Delta}^{1,1}\left(\mathbb{T}, \mathbb{R}^{n}\right) \times W_{\Delta}^{1,1}(\mathbb{T},(0, \infty))$ a solution-tube of (1.1). Then problem (1.1) has a solution $x$ in $W_{\Delta}^{1,1}\left(\mathbb{T}, \mathbb{R}^{n}\right)$ such that $\| x(t)$ $v(t) \| \leq r(t)$ for every $t \in \mathbb{T}$.

In order to establish our multiplicity result, we introduce the notion of a strict solutiontube of (1.1) which will permit one to obtain solutions satisfying $\|x(t)-v(t)\|<r(t)$ for all $t \in[0,1]$.

Definition 3.3 Let $(v, r) \in W_{\Delta}^{1,1}\left(\mathbb{T}, \mathbb{R}^{n}\right) \times W_{\Delta}^{1,1}(\mathbb{T},(0, \infty))$. We say that $(v, r)$ is a strict solution-tube of (1.1) if the following conditions hold:

(i) there exists a l.s.c. mapping $\varepsilon: \mathbb{T} \rightarrow(0, \infty)$ with $\varepsilon(t)<r(t)$ for every $t \in \mathbb{T}$, and such that for $\Delta$-a.e. $t \in \mathbb{T}_{0}$ and all $x \in \mathbb{R}^{n}$ satisfying

$$
r(\sigma(t))-\varepsilon(\sigma(t))<\|x-v(\sigma(t))\| \leq r(\sigma(t))
$$

there exists $y \in F(t, x)$ such that

$$
\left\langle x-v(\sigma(t)), y-v^{\Delta}(t)\right\rangle \leq r^{\Delta}(t)\|x-v(\sigma(t))\| ;
$$

(ii) $\|v(a)-v(b)\|<r(a)-r(b)$.

Obviously, a strict solution-tube is a solution-tube of (1.1).

Definition 3.4 Let $\left(v_{1}, r_{1}\right)$ and $\left(v_{2}, r_{2}\right)$ be two strict solution-tubes of (1.1). They are said to be compatible if the l.s.c. functions $\varepsilon_{1}$ and $\varepsilon_{2}$ in Definition 3.3(i) can be chosen such that for $\Delta$-a.e. $t \in \mathbb{T}_{0}$ and all $x \in \mathbb{R}^{n}$ satisfying

$$
r_{i}(\sigma(t))-\varepsilon_{i}(\sigma(t))<\left\|x-v_{i}(\sigma(t))\right\|<r_{i}(\sigma(t)) \quad \text { for } i=1,2,
$$

there exists $y \in F(t, x)$ such that

$$
\left\langle x-v_{i}(\sigma(t)), y-v_{i}^{\Delta}(t)\right\rangle \leq r_{i}^{\Delta}(t)\left\|x-v_{i}(\sigma(t))\right\| \quad \text { for } i=1,2 .
$$


Remark 3.5 If $F=\{f\}$ is single-valued, then any two strict solution-tubes of (1.1) are compatible.

Here is our main multiplicity result for the system of first order dynamic inclusions (1.1).

Theorem 3.6 Let $F: \mathbb{T}_{0} \times \mathbb{R}^{n} \rightarrow \mathbb{R}^{n}$ be a $\Delta$-Carathéodory set-valued mapping with nonempty, closed, convex values. Assume the following conditions hold:

(i) there exists $\left(v_{0}, r_{0}\right)$ a solution-tube of (1.1);

(ii) there exist $\left(v_{1}, r_{1}\right)$ and $\left(v_{2}, r_{2}\right)$ two compatible strict solution-tubes of (1.1) such that

(a) $T\left(v_{i}, r_{i}\right) \subset T\left(v_{0}, r_{0}\right)$ for $i=1,2$;

(b) $T\left(v_{1}, r_{1}\right) \cap T\left(v_{2}, r_{2}\right)=\emptyset$.

Then problem (1.1) has at least three distinct solutions $x_{0}, x_{1}, x_{2} \in W_{\Delta}^{1,1}\left(\mathbb{T}, \mathbb{R}^{n}\right)$ such that $x_{j} \in T\left(v_{j}, r_{j}\right)$ and $x_{0} \notin T\left(v_{i}, r_{i}\right)$ for $i=1,2$ and $j=0,1,2$.

To prove this result, we will need four lemmas and we will consider the following modified problems for $j=0,1,2$ :

$$
\begin{aligned}
& x^{\Delta}(t)+x(\sigma(t)) \in F_{j}(t, x(\sigma(t))), \quad \Delta \text {-a.e. } t \in \mathbb{T}_{0}, \\
& x(a)=x(b),
\end{aligned}
$$

where $F_{j}: \mathbb{T}_{0} \times \mathbb{R}^{n} \rightarrow \mathbb{R}^{n}$ are defined as follows:

$$
\begin{aligned}
& F_{0}(t, x)=\bar{x}_{0}(\sigma(t), x)+F\left(t, \bar{x}_{0}(\sigma(t), x)\right) \cap G_{0}(t, x) \cap K_{1}(t, x) \cap K_{2}(t, x), \\
& F_{1}(t, x)=\bar{x}_{1}(\sigma(t), x)+F\left(t, \bar{x}_{1}(\sigma(t), x)\right) \cap G_{1}(t, x) \cap K_{1}(t, x) \cap K_{2}\left(t, \bar{x}_{1}(\sigma(t), x)\right), \\
& F_{2}(t, x)=\bar{x}_{2}(\sigma(t), x)+F\left(t, \bar{x}_{2}(\sigma(t), x)\right) \cap G_{2}(t, x) \cap K_{1}\left(t, \bar{x}_{2}(\sigma(t), x)\right) \cap K_{2}(t, x) ;
\end{aligned}
$$

with

$$
\begin{aligned}
& G_{j}(t, x)=G_{\left(v_{j}, r_{j}\right)}(t, x), \quad j=0,1,2 ; \\
& K_{j}(t, x)=K_{\left(v_{j}, r_{j}, \varepsilon_{j}\right)}(t, x), \quad j=1,2 ;
\end{aligned}
$$

and

$$
\bar{x}_{j}(t, x)=\bar{x}_{\left(v_{j}, r_{j}\right)}(t, x), \quad j=0,1,2 .
$$

Here $G_{(v, r)}, K_{(v, r, \varepsilon)}$, and $\bar{x}_{(v, r)}$ are defined in (2.4), (2.5), and (2.6), respectively.

For $j=0,1,2$, we consider $\mathcal{F}_{i}: C\left(\mathbb{T}, \mathbb{R}^{n}\right) \rightarrow C\left(\mathbb{T}, \mathbb{R}^{n}\right)$ defined by

$$
\mathcal{F}_{j}(x)=L\left(\left\{y \in L_{\Delta}^{1}\left(\mathbb{T}_{0}, \mathbb{R}^{n}\right): y(t) \in F_{j}(t, x(\sigma(t))), \Delta \text {-a.e. } t \in \mathbb{T}_{0}\right\}\right),
$$

where $L$ was defined in Proposition 2.7. We show that $\mathcal{F}_{j}$ has nice properties.

Lemma 3.7 Assume the assumptions of Theorem 3.6 hold. Then, for $j=0,1,2, \mathcal{F}_{j}$ is u.s.c., compact with nonempty, convex, compact values. 
Proof Since $\left(v_{1}, r_{1}\right)$ and $\left(v_{2}, r_{2}\right)$ are compatible strict solution-tubes of $(1.1)$, and $\left(v_{0}, r_{0}\right)$ is a solution-tube of (1.1) such that $T\left(v_{0}, r_{0}\right)$ contains $T\left(v_{1}, r_{1}\right) \cup T\left(v_{2}, r_{2}\right)$, one sees that $\Delta$-a.e. $t \in \mathbb{T}_{0}$ and for every $x \in C\left(\mathbb{T}, \mathbb{R}^{n}\right)$,

$$
F_{j}(t, x(\sigma(t))) \neq \emptyset, \quad \forall j=0,1,2 .
$$

Using the fact that $F$ is $\Delta$-Carathéodory, the conclusion follows from Propositions 2.12 and 2.13, and Examples 2.10 and 2.11.

Taking into account the existence property of the topological degree, we can deduce that $\left(3.1_{j}\right)$ has a solution.

Lemma 3.8 Assume the assumptions of Theorem 3.6 hold. Then, for $j=0,1,2$, there exists a bounded open set $\Omega_{j} \subset C\left(\mathbb{T}, \mathbb{R}^{n}\right)$ such that the topological degree

$$
\operatorname{deg}\left(\mathrm{id}-\mathcal{F}_{j}, \Omega_{j}\right)=1
$$

In particular, $\left(3.1_{j}\right)$ has a solution in $\Omega_{j}$.

Proof Let $H_{j}:[0,1] \times C\left(\mathbb{T}, \mathbb{R}^{n}\right) \rightarrow C\left(\mathbb{T}, \mathbb{R}^{n}\right)$ be defined by

$$
H_{j}(\lambda, x)=\lambda \mathcal{F}_{j}(x)
$$

The previous lemma implies that $H_{j}$ is an u.s.c., compact set-valued mapping with nonempty, compact, convex values. Hence, there exists a bounded open set $\Omega_{j} \subset C\left(\mathbb{T}, \mathbb{R}^{n}\right)$ such that

$$
H_{j}\left([0,1] \times C\left(\mathbb{T}, \mathbb{R}^{n}\right)\right) \subset \Omega_{j}
$$

The topological degree theory implies that

$$
\begin{aligned}
1 & =\operatorname{deg}\left(\mathrm{id}, \Omega_{j}\right)=\operatorname{deg}\left(\mathrm{id}-H_{j}(0, \cdot), \Omega_{j}\right)=\operatorname{deg}\left(\mathrm{id}-H_{j}(1, \cdot), \Omega_{j}\right) \\
& =\operatorname{deg}\left(\mathrm{id}-\mathcal{F}_{j}, \Omega_{j}\right) .
\end{aligned}
$$

Thus, $\left(3.1_{j}\right)$ has a solution by the existence property of the topological degree.

In the next lemma, we obtain information on the localization of solutions of $\left(3.1_{j}\right)$.

Lemma 3.9 Under the assumptions of Theorem 3.6, if, for some $j \in\{0,1,2\}, x \in W_{\Delta}^{1,1}\left(\mathbb{T}, \mathbb{R}^{n}\right)$ is a solution of $\left(3.1_{j}\right)$, then $x \in T\left(v_{j}, r_{j}\right)$.

Proof Let $x \in W_{\Delta}^{1,1}\left(\mathbb{T}, \mathbb{R}^{n}\right)$ be a solution of $\left(3.1_{j}\right)$. So, there exists $u \in L_{\Delta}^{1}\left(\mathbb{T}_{0}, \mathbb{R}^{n}\right)$ such that

$$
x^{\Delta}(t)+x(\sigma(t))=\bar{x}_{j}(\sigma(t), x(\sigma(t)))+u(t) \in F_{j}(t, x(\sigma(t))), \quad \Delta \text {-a.e. } t \in \mathbb{T}_{0}
$$


Since $\left(v_{j}, r_{j}\right)$ is a solution-tube of $(1.1)$ and $u(t) \in G_{j}(t, x(\sigma(t)))$, $\Delta$-a.e. $t \in \mathbb{T}_{0}$, one has

$$
\begin{gathered}
\left\langle x(\sigma(t))-v_{j}(\sigma(t)), u(t)-v_{j}^{\Delta}(t)\right\rangle \leq r_{j}^{\Delta}(t)\left\|x(\sigma(t))-v_{j}(\sigma(t))\right\|, \\
\Delta \text {-a.e. on }\left\{t \in \mathbb{T}_{0}:\left\|x(\sigma(t))-v_{j}(\sigma(t))\right\|>r_{j}(\sigma(t))\right\} .
\end{gathered}
$$

Observe that, for $t \in R_{\mathbb{T}}$,

$$
\begin{aligned}
\left\|x(t)-v_{j}(t)\right\|^{\Delta} & =\frac{\left\|x(\sigma(t))-v_{j}(\sigma(t))\right\|-\left\|x(t)-v_{j}(t)\right\|}{\mu(t)} \\
& \leq \frac{\left\langle x(\sigma(t))-v_{j}(\sigma(t)), x(\sigma(t))-v_{j}(\sigma(t))-\left(x(t)-v_{j}(t)\right)\right\rangle}{\mu(t)\left\|x(\sigma(t))-v_{j}(\sigma(t))\right\|} \\
& =\frac{\left\langle x(\sigma(t))-v_{j}(\sigma(t)), x^{\Delta}(t)-v_{j}^{\Delta}(t)\right\rangle}{\left\|x(\sigma(t))-v_{j}(\sigma(t))\right\|} ;
\end{aligned}
$$

and for $\Delta$-almost every $t \in \mathbb{T}_{0} \backslash R_{\mathbb{T}}$,

$$
\begin{aligned}
\left\|x(t)-v_{j}(t)\right\|^{\Delta} & =\frac{\left\langle x(t)-v_{j}(t), x^{\Delta}(t)-v_{j}^{\Delta}(t)\right\rangle}{\left\|x(t)-v_{j}(t)\right\|} \\
& =\frac{\left\langle x(\sigma(t))-v_{j}(\sigma(t)), x^{\Delta}(t)-v_{j}^{\Delta}(t)\right\rangle}{\left\|x(\sigma(t))-v_{j}(\sigma(t))\right\|} .
\end{aligned}
$$

Let $s(t)=\left\|x(t)-v_{j}(t)\right\|-r_{j}(t)$. Combining (3.9), (3.10), (3.11), and (3.12), we deduce that $\Delta$-a.e. on $\left\{t \in \mathbb{T}_{0}: s(\sigma(t))>0\right\}$,

$$
\begin{aligned}
s^{\Delta}(t) \leq & \frac{\left\langle x(\sigma(t))-v_{j}(\sigma(t)), x^{\Delta}(t)-v_{j}^{\Delta}(t)\right\rangle}{\left\|x(\sigma(t))-v_{j}(\sigma(t))\right\|}-r_{j}^{\Delta}(t) \\
= & \frac{\left\langle x(\sigma(t))-v_{j}(\sigma(t)), \bar{x}_{j}(\sigma(t), x(\sigma(t)))-x(\sigma(t))\right\rangle}{\left\|x(\sigma(t))-v_{j}(\sigma(t))\right\|} \\
& +\frac{\left\langle x(\sigma(t))-v_{j}(\sigma(t)), u(t)-v_{j}^{\Delta}(t)\right\rangle}{\left\|x(\sigma(t))-v_{j}(\sigma(t))\right\|}-r_{j}^{\Delta}(t) \\
= & \frac{\left\langle\bar{x}_{j}(\sigma(t), x(\sigma(t)))-v(\sigma(t)), u(t)-v_{j}^{\Delta}(t)\right\rangle}{r_{j}(\sigma(t))} \\
& +r_{j}(\sigma(t))-\left\|x(\sigma(t))-v_{j}(\sigma(t))\right\|-r_{j}^{\Delta}(t) \\
< & \frac{r_{j}(\sigma(t)) r_{j}^{\Delta}(t)}{r_{j}(\sigma(t))}-r_{j}^{\Delta}(t) \\
= & 0 .
\end{aligned}
$$

Taking into account the periodic boundary condition and the fact that $\left(v_{j}, r_{j}\right)$ is a solutiontube of (1.1), we obtain

$$
\begin{aligned}
s(a) & =\left\|x(a)-v_{j}(a)\right\|-r_{j}(a) \\
& \leq\left\|x(b)-v_{j}(b)\right\|-r_{j}(b)+\left\|v_{j}(b)-v_{j}(a)\right\|-r_{j}(a)+r_{j}(b) \\
& \leq s(b) .
\end{aligned}
$$


It follows from (3.13), (3.14), and Lemma 2.6 that $s(t) \leq 0$ for all $t \in \mathbb{T}$. Thus, $x \in$ $T\left(v_{j}, r_{j}\right)$.

The fact that $\left(v_{j}, r_{j}\right)$ is a strict solution-tube of $(1.1)$, when $j \in\{1,2\}$, permits us to get more precision on the localization of the solutions of $\left(3.1_{j}\right)$.

Lemma 3.10 Assume the assumptions of Theorem 3.6 hold. For each $j \in\{1,2\}$, if $x \in$ $T\left(v_{j}, r_{j}\right) \cap W_{\Delta}^{1,1}\left(\mathbb{T}, \mathbb{R}^{n}\right)$ is solution of $\left(3.1_{j}\right)$, then it satisfies

$$
\left\|x(t)-v_{j}(t)\right\|<r_{j}(t) \quad \forall t \in \mathbb{T} .
$$

Proof Let $x \in T\left(v_{j}, r_{j}\right)$ be a solution of $\left(3.1_{j}\right)$ for some $j \in\{1,2\}$. Observe that $\bar{x}_{j}(\sigma(t)$, $x(\sigma(t)))=x(\sigma(t))$ for all $t \in \mathbb{T}_{0}$. Let $u \in L_{\Delta}^{1}\left(\mathbb{T}_{0}, \mathbb{R}^{n}\right)$ be such that

$$
x^{\Delta}(t)+x(\sigma(t))=x(\sigma(t))+u(t) \in F_{j}(t, x(\sigma(t))), \quad \Delta \text {-a.e. } t \in \mathbb{T}_{0}
$$

So, $u \in K_{j}(t, x(\sigma(t))), \Delta$-a.e. $t \in \mathbb{T}_{0}$.

Since $\varepsilon_{j}$ is l.s.c., we can find $\delta_{j}>0$ such that

$$
\delta_{j}<\min \left\{\varepsilon_{j}(t): t \in \mathbb{T}\right\} \quad \text { and } \quad \delta_{j}<r_{j}(a)-r_{j}(b)-\left\|v_{j}(b)-v_{j}(a)\right\|
$$

Observe that the periodic boundary condition and the fact that $\left(v_{j}, r_{j}\right)$ is a strict solutiontube of (1.1) imply that

$$
\begin{aligned}
\left\|x(a)-v_{j}(a)\right\| & \leq\left\|x(b)-v_{j}(b)\right\|-r_{j}(b)+\left\|v_{j}(b)-v_{j}(a)\right\|+r_{j}(b) \\
& <r_{j}(a)-\delta_{j} .
\end{aligned}
$$

Let us suppose that

$$
B=\left\{t \in \mathbb{T}:\left\|x(t)-v_{j}(t)\right\|=r_{j}(t)-\delta_{j}\right\} \neq \emptyset
$$

and $t_{1}=\inf B$. It follows from (3.15) that $t_{1}>a$.

First, we consider the case where $t_{1}$ is not left-scattered; that is $t_{1}=\rho\left(t_{1}\right)$. Since

$$
r_{j}\left(t_{1}\right)-\varepsilon_{j}\left(t_{1}\right)<\left\|x\left(t_{1}\right)-v_{j}\left(t_{1}\right)\right\|=r_{j}\left(t_{1}\right)-\delta_{j}
$$

together with the lower semi-continuity of $\varepsilon_{j}$ and the continuity of $x, v_{j}$ and $r_{j}$, there exists $a \leq t_{0}<t_{1}$ such that

$$
r_{j}(t)-\varepsilon_{j}(t)<\left\|x(t)-v_{j}(t)\right\|<r_{j}(t)-\delta_{j} \quad \text { for all } t \in\left[t_{0}, t_{1}\right)
$$

By the definition of $K_{j}$, one has

$$
\begin{aligned}
\frac{\left\langle x(\sigma(t))-v_{j}(\sigma(t)), x^{\Delta}(t)-v_{j}^{\Delta}(t)\right\rangle}{\left\|x(\sigma(t))-v_{j}(\sigma(t))\right\|} & =\frac{\left\langle x(\sigma(t))-v_{j}(\sigma(t)), u(t)-v_{j}^{\Delta}(t)\right\rangle}{\left\|x(\sigma(t))-v_{j}(\sigma(t))\right\|} \\
& \leq r_{j}^{\Delta}(t), \quad \Delta \text {-a.e. } t \in\left[t_{0}, t_{1}\right) \cap \mathbb{T} .
\end{aligned}
$$


This inequality combined with (3.11) and (3.12) implies that

$$
\begin{aligned}
\left(r_{j}\left(t_{1}\right)-\delta_{j}\right)-\left(r_{j}\left(t_{0}\right)-\delta_{j}\right) & <\left\|x\left(t_{1}\right)-v_{j}\left(t_{1}\right)\right\|-\left\|x\left(t_{0}\right)-v_{j}\left(t_{0}\right)\right\| \\
& =\int_{\left[t_{0}, t_{1}\right) \cap \mathbb{T}}\|x(t)-v(t)\|^{\Delta} \Delta t \\
& \leq \int_{\left[t_{0}, t_{1}\right) \cap \mathbb{T}} \frac{\left\langle x(\sigma(t))-v_{j}(\sigma(t)), x^{\Delta}(t)-v_{j}^{\Delta}(t)\right\rangle}{\left\|x(\sigma(t))-v_{j}(\sigma(t))\right\|} \Delta t \\
& \leq \int_{\left[t_{0}, t_{1}\right) \cap \mathbb{T}} r_{j}^{\Delta}(t) \Delta t \\
& =r_{j}\left(t_{1}\right)-r_{j}\left(t_{0}\right) .
\end{aligned}
$$

This is a contradiction.

Now, we consider the case where $t_{1}$ is left-scattered; that is $t_{1}=\sigma\left(t_{0}\right)>t_{0}$ for some $t_{0} \in \mathbb{T}$. Using (3.11) and the definition of $K_{j}$, we deduce that

$$
\begin{aligned}
\left(r_{j}\left(t_{1}\right)-\delta_{j}\right)-\left(r_{j}\left(t_{0}\right)-\delta_{j}\right) & <\left\|x\left(t_{1}\right)-v_{j}\left(t_{1}\right)\right\|-\left\|x\left(t_{0}\right)-v_{j}\left(t_{0}\right)\right\| \\
& =\mu\left(t_{0}\right)\left\|x\left(t_{0}\right)-v_{j}\left(t_{0}\right)\right\|^{\Delta} \\
& \leq \frac{\mu\left(t_{0}\right)\left\langle x\left(\sigma\left(t_{0}\right)\right)-v_{j}\left(\sigma\left(t_{0}\right)\right), x^{\Delta}\left(t_{0}\right)-v_{j}^{\Delta}\left(t_{0}\right)\right\rangle}{\left\|x\left(\sigma\left(t_{0}\right)\right)-v_{j}\left(\sigma\left(t_{0}\right)\right)\right\|} \\
& =\frac{\mu\left(t_{0}\right)\left\langle x\left(\sigma\left(t_{0}\right)\right)-v_{j}\left(\sigma\left(t_{0}\right)\right), u\left(t_{0}\right)-v_{j}^{\Delta}\left(t_{0}\right)\right\rangle}{\left\|x\left(\sigma\left(t_{0}\right)\right)-v_{j}\left(\sigma\left(t_{0}\right)\right)\right\|} \\
& \leq \mu\left(t_{0}\right) r_{j}^{\Delta}\left(t_{0}\right) \\
& =r\left(t_{1}\right)-r\left(t_{0}\right) .
\end{aligned}
$$

This is a contradiction.

We can conclude that $\left\|x(t)-v_{j}(t)\right\|<r_{j}(t)-\delta_{j}$ for every $t \in \mathbb{T}$.

We are now ready to prove Theorem 3.6.

Proof of Theorem 3.6 For $j=0,1,2$, let $\mathcal{F}_{j}: C\left(\mathbb{T}, \mathbb{R}^{n}\right) \rightarrow C\left(\mathbb{T}, \mathbb{R}^{n}\right)$ be defined in (3.8). From the definition and Proposition 2.7, we see that a fixed point of $\mathcal{F}_{j}$ is a solution of $\left(3.1_{j}\right)$. It follows from Lemma 3.8 that there exists an open bounded set $\Omega_{j} \subset C\left(I, \mathbb{R}^{n}\right)$ such that

$$
\operatorname{deg}\left(\mathrm{id}-\mathcal{F}_{j}, \Omega_{j}\right)=1 \quad \text { for } j=0,1,2
$$

From the proof of Lemma 3.8, $\Omega_{j}$ can be chosen big enough such that

$$
\Omega=\Omega_{0}=\Omega_{1}=\Omega_{2} \supset T\left(v_{0}, r_{0}\right) \supset T\left(v_{1}, r_{1}\right) \cup T\left(v_{2}, r_{2}\right) .
$$

For $j \in\{1,2\}$, we define the open set

$$
U_{j}=\left\{x \in C\left(\mathbb{T}, \mathbb{R}^{n}\right):\left\|x(t)-v_{j}(t)\right\|<r_{j}(t) \text { for all } t \in \mathbb{T}\right\}
$$


Lemmas 3.9 and 3.10, (3.16), and the excision property of the degree imply that

$$
\operatorname{deg}\left(\mathrm{id}-\mathcal{F}_{j}, U_{j}\right)=\operatorname{deg}\left(\mathrm{id}-\mathcal{F}_{j}, \Omega\right)=1 \quad \text { for } j=1,2
$$

$$
\text { For } j \in\{1,2\} \text {, since } x \in T\left(v_{j}, r_{j}\right) \subset T\left(v_{0}, r_{0}\right) \text {, }
$$

$$
\forall x \in \bar{U}_{j}, \quad x(\sigma(t))=\bar{x}_{0}(\sigma(t), x(\sigma(t)))=\bar{x}_{j}(\sigma(t), x(\sigma(t))) \quad \forall t \in \mathbb{T} .
$$

So,

$$
F_{0}(t, x(\sigma(t)))=F_{j}(t, x(\sigma(t))), \quad \Delta \text {-a.e. } t \in \mathbb{T}_{0},
$$

and hence,

$$
\forall x \in \bar{U}_{j}, \quad \mathcal{F}_{0}(x)=\mathcal{F}_{j}(x)
$$

Therefore,

$$
\operatorname{deg}\left(\mathrm{id}-\mathcal{F}_{0}, U_{j}\right)=\operatorname{deg}\left(\mathrm{id}-\mathcal{F}_{j}, U_{j}\right) \quad \text { for } j=1,2
$$

The addition property of the degree, combined with (3.16), (3.17), and (3.18) imply that

$$
\begin{aligned}
& \operatorname{deg}\left(\mathrm{id}-\mathcal{F}_{0}, \Omega \backslash\left(\overline{U_{1} \cup U_{2}}\right)\right) \\
& \quad=\operatorname{deg}\left(\mathrm{id}-\mathcal{F}_{0}, \Omega\right)-\left(\operatorname{deg}\left(\mathrm{id}-\mathcal{F}_{0}, U_{1}\right)+\operatorname{deg}\left(\mathrm{id}-\mathcal{F}_{0}, U_{2}\right)\right) \\
& \quad=\operatorname{deg}\left(\mathrm{id}-\mathcal{F}_{0}, \Omega\right)-\left(\operatorname{deg}\left(\mathrm{id}-\mathcal{F}_{1}, U_{1}\right)+\operatorname{deg}\left(\mathrm{id}-\mathcal{F}_{2}, U_{2}\right)\right) \\
& \quad=1-(1+1) .
\end{aligned}
$$

So, for $j=1,2$, problem $\left(3.1_{j}\right)$ has a solution $x_{j} \in T\left(v_{j}, r_{j}\right)$, and, for $j=0,\left(3.1_{j}\right)$ has a solution

$$
x_{0} \notin T\left(v_{0}, r_{0}\right) \backslash\left(T\left(v_{1}, r_{1}\right) \cup T\left(v_{2}, r_{2}\right)\right) .
$$

Assumption (ii)(b) of Theorem 3.6 implies that $x_{0}, x_{1}$, and $x_{2}$ are distinct.

Finally, for $j=0,1,2$, it follows from the definition of $F_{j}$ that

$$
F_{j}\left(t, x_{j}(\sigma(t))\right) \subset x(\sigma(t))+F\left(t, x_{j}(\sigma(t))\right), \quad \Delta \text {-a.e. } t \in \mathbb{T}_{0} .
$$

We conclude that $x_{0}, x_{1}$, and $x_{2}$ are three distinct solutions of (1.1).

We obtain the following corollary for a real-valued nonlinearity.

Corollary 3.11 For $i=1,2$, let $\alpha_{i}, \beta_{i} \in W_{\Delta}^{1,1}\left(\mathbb{T}_{0}, \mathbb{R}\right)$, and let $F: \mathbb{T}_{0} \times \mathbb{R} \rightarrow \mathbb{R}$ be a $\Delta$ Carathéodory set-valued mapping with nonempty, closed, convex values. Assume the following conditions hold:

(i) $\alpha_{1}(t)<\beta_{1}(t) \leq \beta_{2}(t)$ and $\alpha_{1}(t) \leq \alpha_{2}(t)<\beta_{2}(t)$ for all $t \in \mathbb{T}$;

(ii) there exists $t \in \mathbb{T}$ such that $\beta_{1}(t)<\alpha_{2}(t)$; 
(iii) $\alpha_{i}(a)<\alpha_{i}(b)$ and $\beta_{i}(a)>\beta_{i}(b)$ for $i=1,2$;

(iv) for $i=1,2$, there exists a l.s.c. mapping $\epsilon_{i}: \mathbb{T} \rightarrow(0, \infty)$ such that:

(1) $F(t, x) \cap\left[\alpha_{i}^{\Delta}(t), \infty\right) \neq \emptyset$ for $\Delta$-a.e. $t \in \mathbb{T}_{0}$ and all $x \in \mathbb{R}$ such that $\alpha_{i}(t) \leq x<\alpha_{i}(t)+\epsilon_{i}(t)$

(2) $F(t, x) \cap\left(-\infty, \beta_{i}^{\Delta}(t)\right] \neq \emptyset$ for $\Delta$-a.e. $t \in \mathbb{T}_{0}$ and all $x \in \mathbb{R}$ such that $\beta_{i}(t) \geq x>\beta_{i}(t)-\epsilon_{i}(t)$

(v) (1) there exists $y \in F(t, x)$ such that $y \geq \max \left\{\alpha_{1}^{\Delta}(t), \alpha_{2}^{\Delta}(t)\right\}$ for $\Delta$-a.e. $t \in \mathbb{T}_{0}$ and all $x \in\left(\alpha_{1}(t), \alpha_{1}(t)+\epsilon_{1}(t)\right) \cap\left(\alpha_{2}(t), \alpha_{2}(t)+\epsilon_{2}(t)\right) ;$

(2) there exists $y \in F(t, x)$ such that $y \leq \min \left\{\beta_{1}^{\Delta}(t), \beta_{2}^{\Delta}(t)\right\}$ for $\Delta$-a.e. $t \in \mathbb{T}_{0}$ and all $x \in\left(\beta_{1}(t)-\epsilon_{1}(t), \beta_{1}(t)\right) \cap\left(\beta_{2}(t)-\epsilon_{2}(t), \beta_{2}(t)\right) ;$

(3) for $i, j \in\{1,2\}$ with $i \neq j$, there exists $y \in F(t, x)$ such that $\alpha_{j}^{\Delta}(t) \leq y \leq \beta_{i}^{\Delta}(t)$ for $\Delta$-a.e. $t \in \mathbb{T}_{0}$ and all $x \in\left(\beta_{i}(t)-\epsilon_{i}(t), \beta_{i}(t)\right) \cap\left(\alpha_{j}(t), \alpha_{j}(t)+\epsilon_{j}(t)\right)$.

Then problem (1.1) has at least three distinct solutions $x_{0}, x_{1}, x_{2} \in W_{\Delta}^{1,1}\left(\mathbb{T}_{0}, \mathbb{R}\right)$ such that $\alpha_{1}(t) \leq x_{0}(t) \leq \beta_{2}(t), \alpha_{1}(t)<x_{1}(t)<\beta_{1}(t)$, and $\alpha_{2}(t)<x_{2}(t)<\beta_{2}(t)$ for every $t \in \mathbb{T}$ and $\beta_{1}\left(t_{0}\right) \leq x_{0} \leq \alpha_{2}\left(t_{0}\right)$ for some $t_{0} \in \mathbb{T}$.

Proof Let

$$
v_{0}=\frac{\alpha_{1}+\beta_{2}}{2} \text { and } r_{0}=\frac{\beta_{2}-\alpha_{1}}{2}
$$

and, for $i=1,2$,

$$
v_{i}=\frac{\alpha_{i}+\beta_{i}}{2} \quad \text { and } \quad r_{i}=\frac{\beta_{i}-\alpha_{i}}{2}
$$

Assumptions (i), (iii), and (iv) imply that $\left(v_{0}, r_{0}\right)$ is a solution-tube of (1.1) and $\left(v_{i}, r_{i}\right)$ are strict solution-tubes of (1.1) for $i=1,2$. It follows from (v) that $\left(v_{1}, r_{1}\right)$ and $\left(v_{2}, r_{2}\right)$ are compatible strict solution-tubes. Theorem 3.6 gives the conclusion.

Remark 3.12 In the particular case where $F$ is single-valued, condition (v) of the previous corollary can be omitted.

\section{Competing interests}

The authors declare that they have no competing interests.

\section{Authors' contributions}

All authors contributed equally to the writing of this paper. All authors read and approved the final manuscript.

\section{Author details}

${ }^{1}$ Département de Mathématiques et de Statistique, Université de Montréal, Montréal, H3C 3J7, Canada. ${ }^{2}$ Department of Mathematics, Fasa University, Fars, Iran.

\section{Acknowledgements}

The second author was partially supported by NSERC Canada.

Received: 21 June 2015 Accepted: 2 October 2015 Published online: 16 October 2015

\section{References}

1. Hilger, S: Analysis on measure chains - a unified approach to continuous and discrete dynamical systems. Results Math. 18, 18-56 (1990)

2. Atici, FM, Biles, DC: First order dynamic inclusions on time scales. J. Math. Anal. Appl. 292, 222-237 (2004)

3. Dai, Q, Tisdell, CC: Existence of solutions to first-order dynamic boundary value problems. Int. J. Difference Equ. 1, 1-17 (2006)

4. Diblík, J, Ružicková, M, Šmarda, Z: Wazewski's method for systems of dynamic equations on time scales. Nonlinear Anal. 71, e1124-e1131 (2009) 
5. Gilbert, H: Existence theorems for first order equations on time scales with $\Delta$-Carathéodory functions. Adv. Differ. Equ. 2010, Article ID 650827 (2010)

6. Sun, J-P, Li, W-T: Positive solution for system of nonlinear first-order PBVPs on time scales. Nonlinear Anal. 62, 131-139 (2005)

7. Girejko, E, Torres, DFM: The existence of solutions for dynamic inclusions on time scales via duality. Appl. Math. Lett. $25,1632-1637(2012)$

8. Santos, ILD, Silva, GN: Absolute continuity and existence of solutions to dynamic inclusions in time scales. Math. Ann. 356, 373-399 (2013)

9. Frigon, M, Gilbert, H: Systems of first order inclusions on time scales. Topol. Methods Nonlinear Anal. 37, 147-163 (2011)

10. Sun, J-P, Li, W-T: Existence of positive solutions to semipositone Dirichlet BVPs on time scales. Dyn. Syst. Appl. 16, 571-578 (2007)

11. Frigon, M, Lotfipour, M: Multiplicity results for systems of first order differential inclusions. J. Nonlinear Convex Anal. $16,1025-1040(2015)$

12. Borisovich, YG, Gel'man, BD, Myshkis, AD, Obukhovskii, W: Multivalued mappings. J. Sov. Math. 24, 719-791 (1982): Transl. from Itogi Nauki i Tekhn. Ser. Mat. Anal. 19, 127-230 (1982)

13. Himmelberg, CJ: Measurable relations. Fundam. Math. 87, 53-72 (1975)

14. Hu, S, Papageorgiou, NS: Handbook of Multivalued Analysis, vol. 1. Kluwer Academic, Dordrecht (1997)

15. Deimling, K: Nonlinear Functional Analysis. Springer, Berlin (1985)

16. Cabada, A, Vivero, DR: Expression of the Lebesgue $\Delta$-integral on time scales as a usual Lebesgue integral: application to the calculus of $\Delta$-antiderivatives. Math. Comput. Model. 43, 194-207 (2006)

17. Bohner, M, Peterson, A: Advances in Dynamic Equations on Time Scales. Birkhäuser, Boston (2003)

18. Agarwal, RP, Otero-Espinar, V, Perera, K, Vivero, DR: Basic properties of Sobolev's spaces on time scales. Adv. Differ. Equ. 2006, Article ID 38121 (2006)

19. Frigon, M: Systems of first order differential inclusions with maximal monotone terms. Nonlinear Anal. 66, 2064-2077 (2007)

\section{Submit your manuscript to a SpringerOpen ${ }^{\circ}$ journal and benefit from:}

- Convenient online submission

- Rigorous peer review

- Immediate publication on acceptance

- Open access: articles freely available online

- High visibility within the field

Retaining the copyright to your article 